\title{
Nachruf Dr. Dr. Herbert Falk
}

Unermüdliche Tatkraft, Pioniergeist und unternehmerisches Denken - und vor allem sein außerordentliches Engagement im Bereich der Gastroenterologie und Hepatologie sind Merkmale und Eigenschaften, die das Leben von Dr. Dr. Herbert Falk ausgezeichnet haben.

Der promovierte Pharmazeut und Mediziner übernahm in den 1950er Jahren die väterliche Apotheke in Freiburg i.Br. und begann parallel eine Karriere als pharmazeutischer Unternehmer. Er spezialisierte sich dabei schon früh auf die Hepatologie und die Gastroenterologie.

Mit großem unternehmerischen Geschick und Weitblick baute er die Dr. Falk Pharma GmbH auf und entwickelte sie zu einem national und international erfolgreichen innovativen pharmazeutischen Unternehmen mit Spezialisierung auf der Gastroenterologie und Hepatologie.

Ein wichtiger Meilenstein im beruflichen Leben von Dr. Dr. Herbert Falk war die 1. Falk Leberwoche im Jahre 1967, der nahezu 200 wissenschaftliche internationale Symposien folgen sollten, neben zahlreichen Workshops, gastroenterologischen Foren und ärztlichen Fortbildungsveranstaltungen, die von der von Dr. Dr. Herbert Falk ins Leben gerufenen Falk Foundation e.V. initiiert, organisiert und finanziert wurden. Die Dimension des außergewöhnlichen Firmenkonzeptes, das voll auf die Förderung der Medizin und Wissenschaft setzt, machen am besten Zahlen deutlich: Mehr als 100000 Wissenschaftler aus mehr als 100 Nationen haben an den internationalen Symposien teilgenommen und in den mehr als 11000 Workshops und Fortbildungsveranstaltungen wurden mehr als eine Million Teilnehmer zu aktuellen Themen der Gastroenterologie und Hepatologie geschult. In besonderem Maße hat sich der engagierte Unternehmer dabei der Förderung des wissenschaftlichen Nachwuch- ses verschrieben, wie nicht zuletzt die zahlreichen von der Falk Foundation e.V. ausgelobten Förderpreise und Wissenschaftspreise dokumentieren.

Von der hohen Reputation, die Dr. Dr. Herbert Falk mit diesem außerordentlichen Engagement für die Medizin, die ärztliche Fortbildung und die Wissenschaft erlangte, zeugen zahlreiche nationale und internationale Ehrungen, die er erhielt: Er war Ehrenmitglied in zahlreichen Vereinigungen und Fachgesellschaften, die damit sein enormes Engagement für die Wissenschaft und die ärztliche Kunst würdigten. Dr. Dr. Herbert Falk erhielt die Ehrendoktorwürde der Universitäten Basel und Cluj und wurde 1977 mit dem Bundesverdienstkreuz ausgezeichnet. Im Jahre 2004 erhielt Dr. Dr. Herbert Falk den «AGA Lifetime Distinguished Service Award», eine Auszeichnung für lebenslange außerordentliche Leistungen, die nur zweimal in 10 Jahren durch die Amerikanische Gesellschaft für Gastroenterologie verliehen wurde. Die Ehrung erfolgte als Anerkennung der jahrzehntelangen Förderung der Gastroenterologie durch Dr. Dr. Herbert Falk - ebenfalls ein Meilenstein im Leben des Pharmazeuten und Mediziners, mit dem sein Lebenswerk gewürdigt wurde.

Trotz der hohen internationalen Reputation hat Dr. Dr. Herbert Falk nie seine Wurzeln vergessen. Er war seiner Heimat, dem Badenland, aufs Tiefste verbunden, das Wandern im Schwarzwald, das er in Wanderführern dokumentierte, gab ihm bis ins hohe Alter Entspannung und Kraft.

Er verstarb im Alter von 84 Jahren am 8. August 2008 in seiner Heimatstadt Freiburg i.Br. und wird - nicht zuletzt wegen seines unermüdlichen Einsatzes für die Wissenschaft, seiner Großzügigkeit, seiner Menschlichkeit und seiner beeindruckenden Persönlichkeit - seinen Weggefährten unvergessen bleiben.

Christine Vetter, Köln

\section{KARGER}

(c) 2008 S. Karger GmbH, Freiburg 